\title{
Pédagogie Active Et Développement Des Compétences Entrepreneuriales Des Étudiants : Analyse Empirique
}

\author{
Said Radi (Enseignant chercheur)
}

Mohammed V University in Rabat (UM5R), Morocco

Faculté des sciences juridiques économiques et sociales - Souissi

doi: 10.19044/esj.2017.v13n26p315 URL:http://dx.doi.org/10.19044/esj.2017.v13n26p315

\begin{abstract}
In order to enable the Moroccan University to provide the country with human resources capable of contributing to its development, a reform of higher education was implemented as from 2003. This reform focuses much more on the development of students' skills than on the acquisition of knowledge.

In this context, the UM5R has set up a Specialized Master in Entrepreneurship and Project Management. The main innovation of this training is the introduction of active pedagogy through two activities: entrepreneurial missions and creativity workshops.

This article attempts to answer the question of the extent to which the introduction of this educational innovation has improved the entrepreneurial skills of the students. The chosen research methodology is action research.

The main result is that the introduction of active pedagogy has enabled students to effectively develop entrepreneurial skills, to file patents and to create their own companies.
\end{abstract}

Keywords : Active pedagogy, Entrepreneurial skills, students, missions. creativity

\section{Résumé}

Afin de permettre à l'Université marocaine de doter le pays de ressources humaines capables de contribuer à son développement, une réforme de l'enseignement supérieur a été mise en œuvre à partir de l'année 2003. Cette réforme vise beaucoup plus le développement des compétences des étudiants que l'acquisition des connaissances.

Dans ce cadre, l'UM5R a mis en place un Master Spécialisé «Entrepreneuriat et Management des Projets ». La principale innovation de cette formation est l'introduction de la pédagogie active à travers deux activités : les missions entrepreneuriales et les ateliers de créativité. 
Le présent article cherche à répondre à la problématique de savoir dans quelle mesure l'introduction de cette innovation pédagogique a-t-elle permis d'améliorer les compétences entrepreneuriales des étudiants ? La méthodologie de recherche retenue est la recherche action.

Le résultat principal est que l'introduction de la pédagogie active a permis aux étudiants de développer effectivement des compétences entrepreneuriales, de déposer des brevets et de créer leurs propres entreprises.

Mots clés: Pédagogie active, compétences entrepreneuriales, étudiants, missions, créativité

\section{Introduction}

Le système d'enseignement marocain était basé sur une approche pédagogique traditionnelle et centré sur l'enseignant. Dans la perspective de revaloriser la formation universitaire et permettre aux universités de doter le pays de ressources humaines capables de contribuer à son développement économique et social, une réforme de l'enseignement supérieur a été mise en œuvre à partir de l'année 2003. Les principaux objectifs de cette réforme sont :

- orienter le système de l'enseignement beaucoup plus vers le développement des compétences que l'acquisition des connaissances ;

- développer des capacités méthodologiques, linguistiques et de communication ;

- améliorer le rendement du système pédagogique.

Pour atteindre ces objectifs, une nouvelle architecture du système d'enseignement a été envisagée. Il s'agit d'une organisation des études en cycles, semestres, filières et modules. Ainsi, la nouvelle approche pédagogique est basée essentiellement sur le système modulaire.

A cet égard, il convient de rappeler que dans la conception d'une formation, deux modèles pédagogiques peuvent être distingués :

- Le premier modèle, centré sur les savoirs à transmettre, est relatif à une pédagogie de contenu où enseigner est avant tout transmettre un savoir. L'enseignant est placé donc au centre du système éducatif.

- Le second modèle, basé sur une pédagogie des compétences, consiste à développer chez l'apprenant les compétences requises dans les trois domaines du savoir que sont les connaissances, les habilités et les attitudes. Le système, cette fois ci, est centré sur ce que l'étudiant sera capable de faire et d'exercer à l'issue de sa formation.

Ainsi, selon la nouvelle approche pédagogique, l'enseignement ne se limite pas à définir un ensemble de connaissances à véhiculer mais une liste des compétences à développer. Dans le cadre de cette réforme, l'UM5R a 
créé une filière de Master Spécialisé en «Entrepreneuriat et Développement International » (EDI). La principale innovation de cette formation est l'introduction de la pédagogie active à travers deux activités qui se basent sur un «apprentissage Expérientiel»: les missions entrepreneuriales et les ateliers de créativité.

La problématique proposée par le présent article est de savoir dans quelle mesure l'introduction de cette innovation pédagogique a-t-elle permis d'améliorer les compétences entrepreneuriales des étudiants ? La méthodologie de recherche retenue pour répondre à cette problématique est la recherche action.

Avant de développer ladite méthodologie et de présenter les résultats de notre étude, il est jugé nécessaire de situer le cadre théorique de notre recherche.

\section{Cadre théorique de la recherche}

1.1 La pédagogie active

La pédagogie active se réfère historiquement à Adolphe Ferrière qui, au début du XXe siècle (1922), a été parmi les premiers à utiliser l'appellation école active dans ses publications. Dans le courant de cette « nouvelle pédagogie », il est important de citer également des pédagogues comme E. Claparède, John Dewey, Maria Montessori, C. Freinet et Jean Piaget. Le Tableau 1 ci-après synthétise les contributions de chacun de ces auteurs :

Tableau 1. Les fondateurs de la pédagogie active

\begin{tabular}{|c|l|}
\hline $\begin{array}{c}\text { Adolphe Ferrière } \\
(1879-1960)\end{array}$ & $\begin{array}{l}\text { l'Ecole active : Le père de la PA et 1 }{ }^{\text {er }} \text { qui a parlé de l'Ecole active en } \\
1922 .\end{array}$ \\
\hline $\begin{array}{c}\text { E. Claparède } \\
(1873-1940)\end{array}$ & $\begin{array}{l}\text { Adaptation par le tâtonnement : L'élève doit comprendre le sens de } \\
\text { ce qu'il fait. L'enseignant devra éveiller le désir de résoudre un } \\
\text { problème : « A quoi, cela sert-il de savoir? » }\end{array}$ \\
\hline $\begin{array}{c}\text { John Dewey } \\
(1859-1952)\end{array}$ & $\begin{array}{l}\text { Activité centrée sur l'élève : La pédagogie employée doit être fondée } \\
\text { sur l'intérêt de l'élève, son activité lui permettant une continuelle } \\
\text { reconstruction de l'expérience au milieu d'une vie communautaire. }\end{array}$ \\
\hline $\begin{array}{c}\text { Maria } \\
\text { Montessori } \\
(1870-1952)\end{array}$ & $\begin{array}{l}\text { L'expérimentation : L'élève apprend en agissant, en manipulant en } \\
\text { respectant son propre rythme, sa motivation. }\end{array}$ \\
\hline $\begin{array}{c}\text { Jean Piaget } \\
(1896-1980)\end{array}$ & $\begin{array}{l}\text { Constructivisme : Le savoir de l'élève se construit au départ de } \\
\text { l'activité de ce dernier (manuelle ou intellectuelle) }\end{array}$ \\
\hline $\begin{array}{c}\text { C. Freinet } \\
\text { (1896-1966) }\end{array}$ & $\begin{array}{l}\text { Tâtonnement expérimental : En fonction de ses besoins et de ses } \\
\text { centres d'intérêts, l'enfant, au centre de ses apprentissages, va découvrir } \\
\text { le monde par lui-même grâce à l'expérience et donc à la pratique des } \\
\text { essais-erreurs. }\end{array}$ \\
\hline
\end{tabular}

Il y a beaucoup de différences entre ce qu'était la pédagogie active du début du siècle et ce qu'elle est aujourd'hui, car elle a comme particularité d'être en évolution constante. En effet, dans un ouvrage collectif dirigé par 
Raucent et Vander Borght (2006) la pédagogie active est définie comme un ensemble de méthodes qui relève de ce qu'on appelle «L'apprentissage expérientiel », c'est à dire "apprendre en faisant». Elle a pour but de rendre l'apprenant acteur de ses apprentissages, afin qu'il construise ses savoirs à travers des situations de recherche.

Pour mettre en œuvre ce type de pédagogie au sein de la filière EDI de l'UM5R, nous avons expérimenté deux pratiques : Les missions tutorées et les ateliers de créativité. Le modèle théorique retenu dans notre recherche est celui de Marcel LEBRUN (2007) qui peut être schématisé ainsi :

Figure 1. Modèle de Marcel LEBRUN

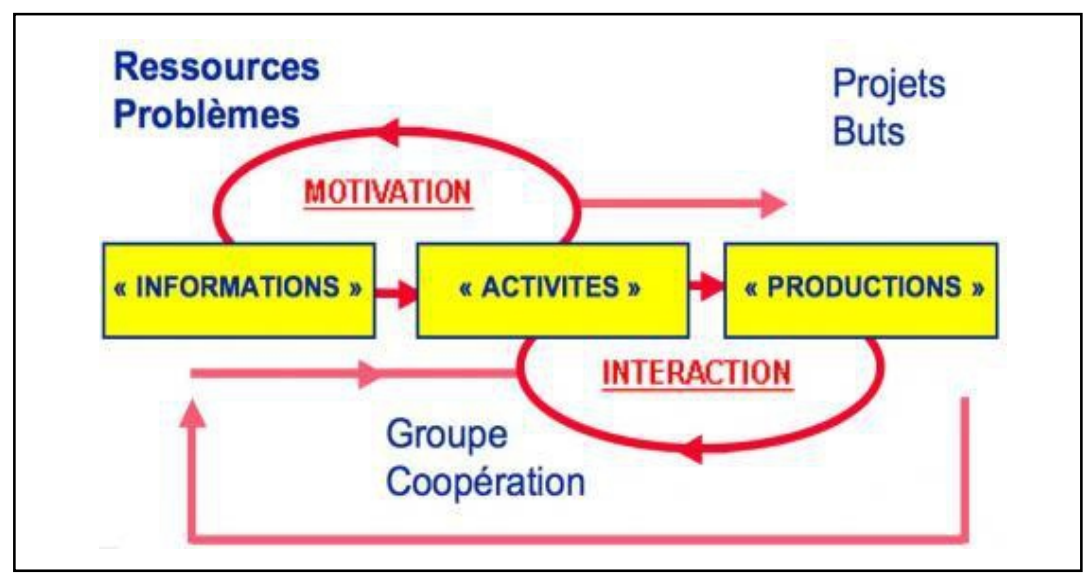

Source : LEBRUN (2007)

Le modèle de Lebrun (2007) se compose de cinq facteurs en interaction qui permettent de caractériser des situations d'enseignement et d'apprentissage orientées vers la pédagogie active.

\subsection{Les compétences visées}

De nombreuses approches ont décomposé la compétence en trois savoirs: des savoirs (connaissances), des savoir-faire (pratique professionnelle) et des savoir-être (comportements, attitudes, capacités d'adaptation). C'est ainsi que pour Jonnaert (2002), la compétence est la mise en œuvre, par une personne particulière ou par un groupe de personnes, de savoirs, de savoir-être, de savoir-faire ou de savoir-devenir dans une situation donnée. Quant à Tardif (2006), il définit la compétence comme un savoir-agir complexe qui prend appui sur la mobilisation et la combinaison efficace d'une variété de ressources internes et externes à l'intérieur d'une famille de situations. Un autre spécialiste de la compétence est allé dans le même sens que Tardif; il s'agit de Guy Le Boterf qui distingue dans son livre «Repenser la compétence» (2010, page 21) : être compétent «c'est être capable d'agir et de réussir avec pertinence dans une 
situation de travail » et avoir des compétences c'est «posséder des ressources pour agir avec compétence »

S'agissant des compétences entrepreneuriales, l'analyse de la documentation scientifique des productions des chercheurs en management montre qu'avant 1990 les chercheurs ont très peu fait allusion aux compétences que doivent posséder les entrepreneurs. Dans les années 90, Hamel et Prahalad vont reprendre la théorie de la ressource (Penrose en 1959) pour montrer que l'entreprise va faire la différence par rapport à une autre par la détention de ressources rares. Ils prennent en compte la dimension stratégique de la compétence ce qui va permettre le développement d'une véritable approche par la compétence (Stéphane Jacquet 2011).

Par ailleurs, au début des années 90, un nouveau courant émerge et met l'accent sur la forte relation entre la performance de l'entreprise et les compétences de l'entrepreneur. Ce courant s'oriente vers la caractérisation de ce dernier par ce qu'il fait et non pas par ce qu'il est (Gartner, 1988). Ainsi, l'accent est résolument placé sur les actions de l'entrepreneur avec en creux ses compétences. Progressivement apparaissent les premiers travaux typologiques visant à classifier les compétences entrepreneuriales et à proposer un référentiel de compétences dont notamment ceux de Chandler et Jansen (1992), Herron et Robinson (1993), Baum (1995), Laviolette et Loue (2006), Boyles (2012).

Parmi les compétences entrepreneuriales classées par les chercheurs ou citées dans les référentiels proposés, nous avons retenu quatre à savoir :

1. Capacité de mobiliser des ressources (Boyles),

2. Capacité à résoudre des problèmes (Boyles),

3. Faire preuve de leadership (Baum, chandler, Laviolette et Loue) et

4. Créer et innover (Laviolette, Loue et Boyles).

Le choix de ces compétences est justifié d'une part par le fait qu'elles sont citées dans plusieurs référentiels de compétences entrepreneuriales et d'autre part par la possibilité de mesurer leur évolution selon les activités proposées aux étudiants.

\section{Méthodologie de recherche : Recherche action}

La méthodologie de recherche jugée la plus adaptée à notre problématique est la recherche action. En effet,

«La recherche-action est un processus destiné à doter tous les participants de la scène éducative, qu'il s'agisse des étudiants, des enseignants ou d'autres intervenants, des moyens d'améliorer leurs pratiques grâce à leurs expériences éclairées et nourries des savoirs théoriques en cours. Tous les participants deviennent acteurs consentants $d u$ processus de recherche. ") (Michel Catroux 2002, page 10). 
L'objectif principal de la recherche-action est de fournir un cadre aux investigations qualitatives effectuées par les enseignants et les chercheurs en situations complexes de classe. Elle permet d'identifier un problème et de chercher sa solution par la mise en place de stratégies visant à l'amélioration d'une situation insatisfaisante pour chacun des apprenants.

Elle est donc adaptée à des situations où il est souhaitable d'appliquer une action pour obtenir un changement et, en même temps, de développer une meilleure compréhension qui permet d'instruire le changement même et d'améliorer une situation concrète.

Selon Lewin (1946) et Susman (1983), le processus de la recherche action est constitué de cinq étapes :

- Indentification du problème ;

- Plan d'action ;

- Mise en œuvre de l'action ;

- Evaluation des effets de l'action et

- Valorisation de la recherche.

Ces étapes sont schématisées dans la Figure 2.

Figure 2. Processus de la Recherche action

Source : Elaboré à partir des travaux de Lewin (1946) et Susman (1983)

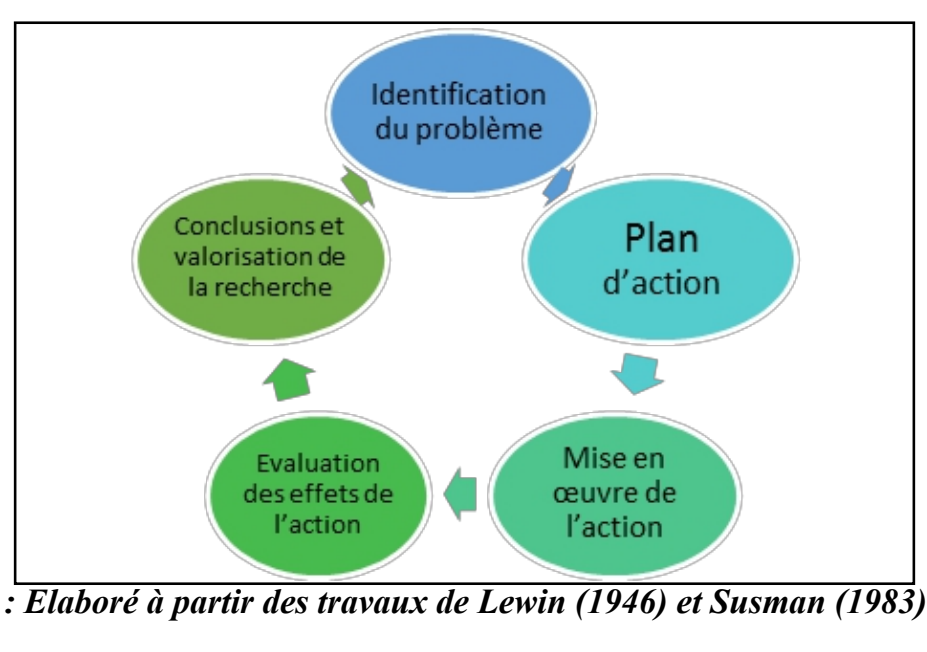

\subsection{Identification du problème}

Les lauréats des établissements à accès ouvert souffrent d'un grand problème de compétences de «Savoir-Faire» et de «Savoir-Etre ». Ce problème est dû, à notre humble avis, aux pratiques pédagogiques adoptées dans ce type d'établissement et à la nature des enseignements dispensés. Ces deux facteurs ne permettent pas aux étudiants de développer de telles compétences. 


\subsection{Plan d'action}

Pour tenter de résoudre le problème ainsi identifié, nous avons procédé à l'introduction de la pédagogie active au niveau du Master EDI et ce, à travers deux activités qui se basent sur un apprentissage expérientiel. Par la suite, nous avons essayé de mesurer l'impact de cette pratique pédagogique sur le développement des compétences des étudiants à travers deux études : une qualitative (les observations et les entretiens) et une autre quantitative (Enquête auprès de 60 étudiants).

\subsection{Mise en ouvre de l'action}

Les deux activités qui ont été expérimentées pour permettre aux étudiants «d'apprendre en faisant» (learning by doing) sont : les missions tutorées et les ateliers de créativité.

\subsubsection{Les missions tutorées}

Au niveau de la filière Master Spécialisé EDI, nous avons prévu un module intitulé " Mission tutorée ». Ce type d'activité est très peu proposé dans les universités marocaines. Ces missions constituent une expérience pédagogique originale. Elles sont organisées à la fin de chacun des trois premiers semestres (S1, S2 et S3).

Sur une durée d'un mois, les étudiants, par groupe de trois ou quatre, travaillent sur une problématique réelle ou un projet proposé par l'organisme partenaire. Chaque groupe d'étudiants est coaché par un tuteur pédagogique, enseignant de l'université, et un tuteur professionnel relevant de l'établissement d'accueil. Généralement c'est ce dernier qui propose le travail demandé aux étudiants.

Les objectifs assignés à la mission ainsi que les résultats attendus sont clairement définis dans « une lettre de mission » en commun accord entre le groupe de travail et le professionnel. Il s'agit de déployer une activité véritable pour une fin précise. Les étudiants agissent comme des consultants juniors. La lettre est remise par la suite au tuteur pédagogique qui peut émettre une opinion sur la faisabilité de la mission. Après sa validation, les étudiants démarrent leur travail.

\section{La réalisation de la mission ou du projet comporte :}

- des difficultés, que l'apprenant doit aplanir ;

- des informations qu'il doit chercher là ou elles se trouvent ;

- des problèmes qu'il doit résoudre ;

- des conflits entre les membres de groupe qu'il doit gérer ;

- des contenus qu'il doit comprendre, définir, assimiler, réutiliser ;

- des plans qu'il doit élaborer et en assurer l'exécution. 
Ainsi, l'étudiant devient lui-même acteur de ses apprentissages, il construit ses savoirs en travaillant sur des situations réelles. Il est certes encadré, mais il a une marge de manœuvre assez large pour être autonome dans sa démarche.

L'apprentissage passe ainsi d'une séquence traditionnelle :

- programmes à suivre ;

- exercices calibrés pour appliquer les notions développées ;

- contrôles pour tester les savoirs et savoir-faire (évaluation)

à une séquence de :

- confrontation à un problème réel ;

- recherche d'information concernant ce problème (autoformation) ;

- recherche d'une solution au problème.

Dans cette activité pédagogique, le rôle de l'enseignant est réduit à l'orientation et l'assistance de l'étudiant qui est l'acteur principal de ce processus d'apprentissage. Le suivi de la mission est assuré à travers des « Fiches de mission » qui sont remplies régulièrement par chaque groupe et envoyées au tuteur pédagogique à des dates fixées dans le guide de mission. L'évaluation de l'activité porte sur la globalité de la démarche. Des fiches d'appréciation sont remplies par les deux tuteurs pour évaluer essentiellement le savoir, le savoir- faire et le savoir-être de l'étudiant. Une soutenance des rapports de mission est prévue avec la participation des universitaires et des professionnels.

\subsubsection{Les ateliers de créativité}

L'un des principaux objectifs de la filière Master EDI est de développer chez l'étudiant l'esprit d'entrepreneuriat et de créativité. A cet égard, une activité d'apprentissage est organisée par l'équipe pédagogique sous forme d'atelier dans lequel les 60 étudiants du Master M1 et M2 sont regroupés ensemble pour chercher des idées innovantes qui peuvent être brevetées. Chaque équipe est renforcée par des étudiants d'autres Masters (Commerce, Marketing, etc.) ainsi que des élèves ingénieurs invités des écoles de l'enseignement technique. L'objectif est de créer des groupes de travail hétérogènes pour optimiser les synergies existantes entre les différentes formations.

Cette activité est encadrée par plusieurs enseignants. Lors de la première séance, sont présentés les objectifs assignés à l'activité, le déroulement des ateliers, la démarche à suivre, les résultats attendus et les modalités d'évaluation. Par ailleurs, un séminaire sur la créativité et la recherche des idées innovantes est animé par un spécialiste. Ensuite, une autre rencontre est prévue pour présenter les premières idées de projets innovants. Entre les deux rencontres, les équipes sont invitées à s'organiser elles-mêmes pour préparer le travail demandé. 
Un premier jury, composé d'enseignants et de professionnels, est constitué pour sélectionner parmi les idées présentées, par chaque groupe, une idée qui pourra être retenue.

La dernière étape de ces ateliers de créativité consiste à inviter des spécialistes de l'Office Marocain de la Propriété Industrielle et Commerciale (OMPIC) pour juger si les idées retenues sont brevetables. Chaque groupe, passe devant le deuxième jury composé des spécialistes, pour présenter son idée avec éventuellement un prototype. A ce stade, le rôle des étudiants informaticiens, chimistes, physiciens devient très important au sein de l'équipe car ils peuvent utiliser les laboratoires de leurs écoles pour préparer les prototypes.

\subsubsection{Le Modèle de Lebrun et les activités proposées}

Comme il a été déjà signalé dans le premier paragraphe, les deux activités ont été expérimentées en s'inspirant du Modèle de Lebrun. Ainsi, on retrouve dans les deux expériences les cinq facteurs proposés par ce modèle (figure 1) :

- Les informations : il s'agit de mettre à la disposition de l'étudiant des ressources variées de la part de l'enseignant et du tuteur professionnel. Par ailleurs, l'étudiant lui-même est invité à chercher et à construire d'autres informations ;

- La motivation: la perception des contextes étant un facteur préliminaire de motivation, les activités proposées sont généralement beaucoup plus contextualisées ;

- Les activités : pour assurer l'implication de l'étudiant et lui permettre de développer ses compétences, les activités proposées sont de type résolution de problème, étude de cas ou mise en projet ;

- Les interactions : les étudiants sont invités à travailler en groupe et les interactions sont plus fréquentes;

- Les productions : le processus d'apprentissage est couronné par des productions que doit partager l'étudiant avec ses pairs, l'enseignant et les partenaires.

\section{$2.4 \quad$ Suivi et évaluation des deux activités}

Rappelons que l'objectif recherché derrière l'introduction la pédagogie active dans la filière EDI est de développer chez les étudiants des compétences entrepreneuriales. Quatre parmi ces compétences recherchées ont été suivies et évaluées (Figure 3) : 
Figure 3. Les compétences évaluées

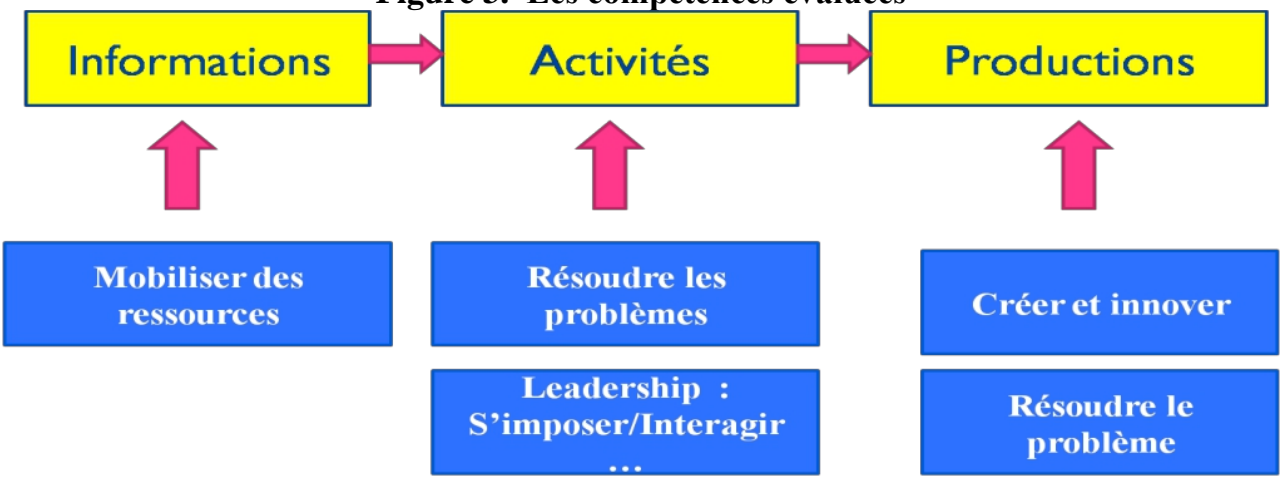

Les évaluations sont faites par les enseignants et les professionnels tout au long du déroulement des activités et pendant les séances d'encadrements et ce, à l'aide des grilles d'observations et d'appréciation. Des évaluations sont réalisées également par les paires.

Les critères d'évaluation utilisés sont résumés dans le Tableau 2. Chaque critère est noté sur 1 point et chaque compétence sur 5 points pour avoir une note finale de 20 points. La même grille d'évaluation est retenue pour les ateliers de créativité et les 3 missions tutorées afin de suivre l'évolution et le développement dans le temps des compétences de l'étudiant.

Tableau 2. Critères d'évaluation des compétences ${ }^{7}$

\begin{tabular}{|c|c|}
\hline Compétences & Critères d'évaluation \\
\hline Mobiliser des ressources & $\begin{array}{l}\text { - Efficacité des stratégies de recherche } \\
\text { - Pertinence des sources consultées } \\
\text { - Cohérence dans l'organisation de l'information } \\
\text { - Capacité à construire des ressources non disponibles. } \\
\text { - Capacité à exploiter les technologies de l'information et de } \\
\text { communication }\end{array}$ \\
\hline Résoudre des problèmes & $\begin{array}{l}\text { - Précision de la définition du problème } \\
\text { - Pertinence des stratégies envisagées } \\
\text { - Qualité du retour sur la démarche } \\
\text { - Utilisation des difficultés et des erreurs de parcours pour } \\
\text { progresser } \\
\text { - Souplesse dans la poursuite des pistes de solution }\end{array}$ \\
\hline Leadership & $\begin{array}{l}\text { - S'imposer en tant que leader } \\
\text { - Susciter l'adhésion et l'implication des collaborateurs } \\
\text { - Capacité de gérer des conflits entre les membres du groupe } \\
\quad-\text { Capacité à communiquer } \\
\text { - Confiance en soi }\end{array}$ \\
\hline Créer et innover & - Diversité des idées générées et des scénarios envisagés \\
\hline
\end{tabular}

7 Ces critères ont été choisis en s'inspirant du programme de formation de l'école québécoise, voir le lien suivant :

www.eduation.gouv.qc.ca/fileadmin/site_web/documents/dpse/formation_jeunes/3-pfeqchap3 


\begin{tabular}{|l|l|}
\hline & - Degré d'ouverture dans l'exploration de nouvelles façons de \\
faire & - Originalité des liens établis entre les éléments d'une situation \\
& - Souplesse dans l'exploitation de nouvelles idées \\
& - Degré de tolérance dans des situations ambigües
\end{tabular}

Il importe de signaler qu'au terme des activités programmées, des entretiens ont été réalisés avec les professionnels pour donner leurs appréciations. Par ailleurs, nous avons jugé opportun d'associer les étudiants à l'évaluation de cette expérience puisqu'ils sont au centre du modèle pédagogique proposé. Pour ce faire, un questionnaire a été administré auprès de 60 étudiants. L'expérimentation des deux pratiques de la pédagogique active et l'adoption de la méthodologie de recherche action nous ont permis d'aboutir à des résultats dont certains seront présentés et discutés dans le paragraphe suivant.

\section{Résultats et discussion}

Le suivi de l'activité « Missions tutorées » pendant les trois semestres a permis de calculer la moyenne de la classe pour chaque type de compétence évaluée. Le graphique 1 suivant illustre l'évolution des compétences visées :

Graphique 1. L'évolution des compétences visées

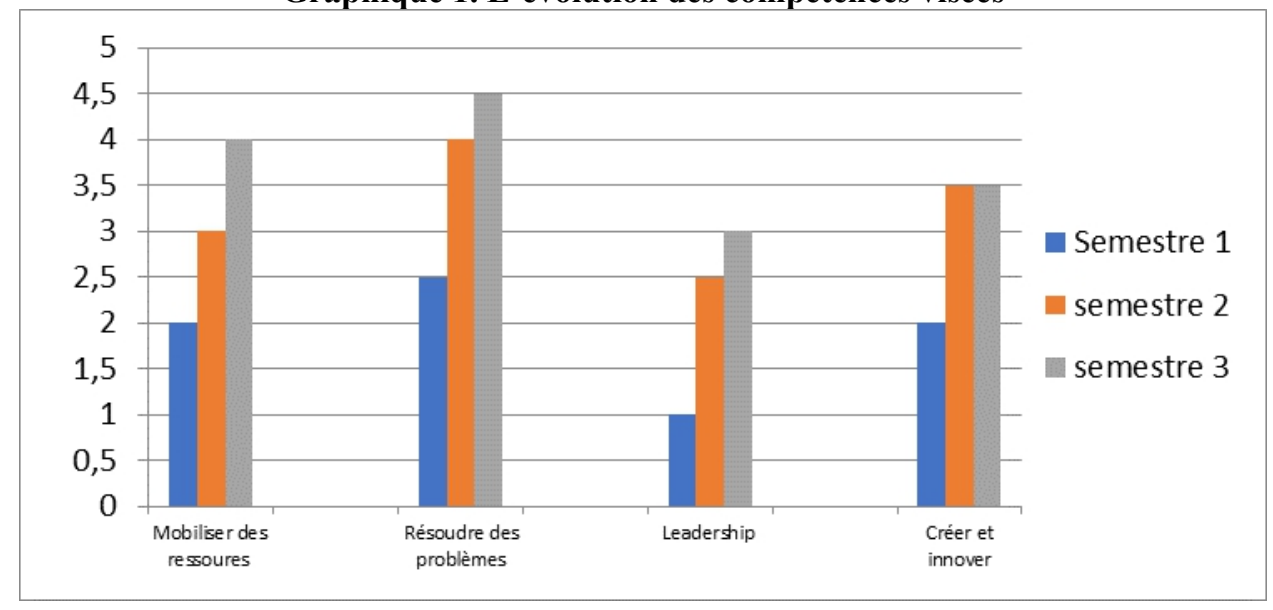

Il importe de remarquer que la principale compétence développée chez les étudiants pendant les 3 semestres est leur capacité à résoudre des problèmes, suivie de la capacité à mobiliser des ressources et à innover. Ces résultats sont confirmés par les entretiens réalisés avec les professionnels qui ont exprimé leur satisfaction de la production des étudiants au terme des missions. Ils ont également témoigné d'une grande capacité des étudiants à chercher et à exploiter l'information. En revanche, la qualité de leadership reste peu présente chez nos étudiants surtout au niveau de la première mission. Rares sont les étudiants qui ont pris l'initiative et essayé d'organiser 
et gérer le travail de leur groupe. Toutefois, une nette amélioration est observée à partir de la deuxième mission.

D'une manière générale, l'expérience des missions tutorées a permis de développer les quatre compétences visées comme le montre le Graphique 2 suivant :

Graphique 2. Progression des compétences évaluées ${ }^{8}$

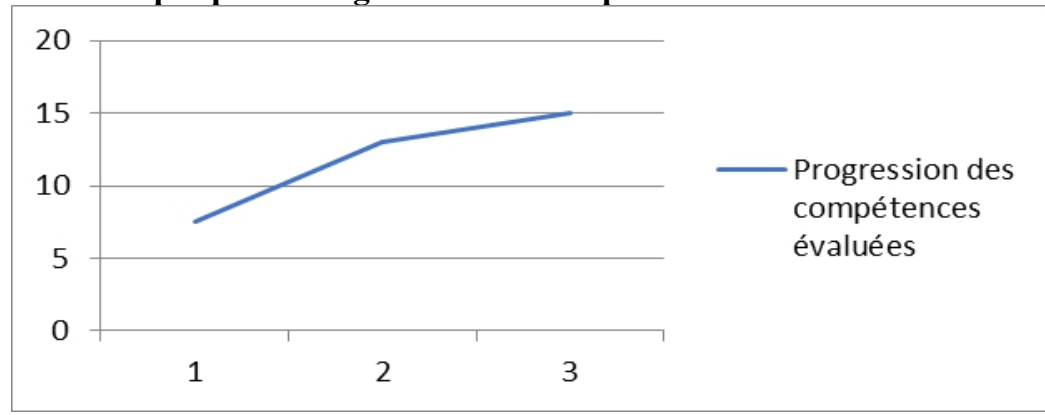

Concernant l'activité des ateliers de créativité, la première compétence développée est la capacité de créativité et d'innovation avec un score de 16/20 suivie de la capacité de résoudre le problème et de mobiliser les ressources (voir graphique 3 La compétence de leadership reste toujours en dernière position. Ce dernier résultat peut être imputable en partie au fait que dans population des étudiants, plus de $60 \%$ sont de sexe féminin. Or, les filles sont généralement timides et prennent du temps pour prendre l'initiative surtout celles qui proviennent du milieu social moyen ou défavorisé comme le cas de notre Faculté.

Graphique 3. Compétences développées au niveau des ateliers de créativités (note/20)

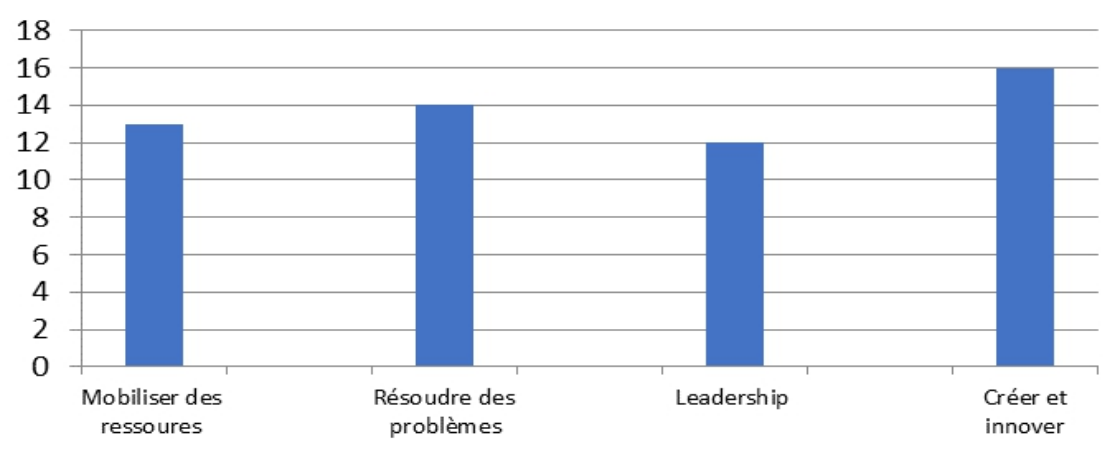

Les résultats réalisés par les ateliers de créativité sont très satisfaisants. En effet, parmi 13 idées de projets innovants présentées, 5 ont été jugées brevetables par les experts de l'OMPIC et 3 ont fait l'objet effectivement de dépôt de brevets.

${ }^{8}$ Cette moyenne a été calculée à partir des moyennes de chaque type de compétences. 
Or, le dépôt de 3 brevets demeure une première dans 1'histoire des Facultés des Sciences Juridiques Economiques et Sociales au Maroc, car ce sont généralement les facultés des sciences exactes et les écoles d'ingénieurs qui déposent le plus souvent des brevets.

Ces résultats témoignent de l'efficacité de l'approche pédagogique adoptée et sa capacité à motiver les apprenants et à les amener à être créatifs.

Par ailleurs, et en vue de faire participer les 60 étudiants à l'évaluation des ces deux activités, ils étaient invités à répondre à un questionnaire élaboré par l'équipe pédagogique. Les principaux résultats de cette enquête sont résumés ainsi :

- $89 \%$ des étudiants jugent que ces activités d'apprentissage sont très importantes ;

- 63\% déclarent avoir bien maitrisé certaines notions ou parties du cours lors de la réalisation de la mission plutôt que lors de l'enseignement du module ;

- $55 \%$ avancent que ces missions leur ont permis de comprendre l'intérêt de certains modules ;

- Concernant l'appréciation par les étudiants des compétences qu'ils ont pu développer grâce à cette nouvelle approche pédagogique, les réponses collectées sont regroupées dans le Tableau 3 suivant :

Tableau 3. Appréciation par les étudiants des compétences développées

\begin{tabular}{|l|c|}
\hline \multicolumn{1}{|c|}{ Compétences développées } & $\begin{array}{c}\text { Classement selon la } \\
\text { Fréquence de citation }\end{array}$ \\
\hline Disposition de travail en groupe & 1 \\
\hline Capacité de résolution de problème & 2 \\
\hline Confiance en soi & 3 \\
\hline Chercher et construire l'information & 4 \\
\hline Créativité et innovation & 5 \\
\hline Leadership & 6 \\
\hline Capacité de communication & 7 \\
\hline Sens de l'organisation & 8 \\
\hline Capacité d'analyse & 9 \\
\hline Savoir négocier & 10 \\
\hline
\end{tabular}

Les jugements des étudiants vont généralement dans le même sens que les évaluations faites par les tuteurs. En effet, les compétences visées par l'étude sont classées parmi les cinq premières sauf pour le leadership qui vient en $6^{\text {ème }}$ position. Ce résultat nous invite à multiplier les pratiques et les méthodes pédagogiques susceptibles de développer chez nos étudiants cette compétence de leadership jugée nécessaire dans le profil d'un entrepreneur.

\section{Conclusion}

L'introduction de la pédagogie active au sein du Master EDI constitue une véritable innovation pédagogique. L'objectif du présent travail était 
d'évaluer les effets de cette innovation sur les compétences entrepreneuriales des étudiants. La méthodologie de recherche que nous avons adoptée, à savoir la recherche action, a permis d'atteindre ledit objectif. Ainsi, l'expérimentation des deux activités des missions tutorées et des ateliers de créativité a contribué efficacement au développement des compétences entrepreneuriales des étudiants et de leur esprit d'analyse et de créativité.

Par ailleurs, les effets positifs de la pédagogie active ne se sont pas fait sentir uniquement chez des étudiants mais également chez les enseignants et les professionnels. En effet, cette pratique pédagogique a permis :

- aux étudiants une progression notable des compétences visées durant les trois semestres et de développer un savoir-faire, un savoir-être, un savoir-devenir et surtout savoir-agir ;

- aux enseignants de découvrir des nouvelles potentialités chez certains étudiants classés très moyens dans l'enseignement des cours et d'avoir un retour sur cette expérience ;

- aux professionnels de profiter de la production des étudiants et changer leur attitude négative vis-à-vis des lauréats des établissements à accès ouvert.

Dans le cadre de l'élargissement de l'expérience de la pédagogie active, il est prévu :

- d'acquérir un logiciel de jeux d'entreprise pour permettre aux étudiants de faire des simulations de cas fictifs et par conséquent de s'éloigner des contextes conflictuels et affectifs de la réalité et de dédramatiser l'apprentissage en s'amusant à faire des choses importantes sans leur donner de l'importance ;

- de préparer des cas réels par certains enseignants en collaboration avec des cadres d'entreprises et de faire travailler les étudiants en groupes sur ces cas.

Nos futurs travaux de recherche poursuivront l'étude de l'impact d'autres méthodes pédagogiques sur le développement des compétences transversales des étudiants. La population cible pourra être élargie pour englober les étudiants d'autres formations telles que la licence professionnelle et la licence fondamentale.

\section{References:}

1. Allal, L. (2007). Evaluation: Lien entre enseignement et apprentissage. Dans V. Dupriez, \& G. Chapelle. (Eds.). Enseigner. (pp. 139-49). Paris : PUF.

2. Anderson, L.W., \& Krathwohl, D. R. (2001). A taxonomy for learning, teaching and assessing : A revision of bloom's taxonomy of educational objectives. New York : Longman. 
3. Bachy, S, Lebrun, M et Smidts, D. (2010). Un modèle-outil pour fonder l'évaluation en pédagogie active : impact d'une formation sur le développement professionnel des enseignants, Revue internationale de pédagogie de l'enseignement supérieur [Online], 26-1 2010, Online since 10 May 2010, date de dernière consultation 22 mars 2017.

4. Biggs, J. B. (1999). Teaching for quality learning at university. Buckingham: The society for Research into High Education and Open University Press.

5. Catroux, M. (2002). Introduction à la recherche-action : modalité d'une démarche théorique centrée sur la pratique, $L a$ revue Recherche et pratiques pédagogiques en langues de spécialité Cahiers de l'APLIUT, Vol XXI N 3/200

6. Deschuyteneer, M. (2010). La pédagogie active ou comment susciter la motivation des élèves. Travail de fin d'études en vue de l'obtention du Certificat d'Aptitudes Pédagogiques. Institut d'enseignement de promotion sociale de la communauté française a Morlanwelz.

7. Jacquet, S. (2011). Le métier d'entrepreneur : des compétences à développer, acquérir et maîtriser, Publications du Centre des Ressources en Economie et Gestion, 23 janvier 2011. (en ligne) www.creg.ac-versailles.fr/le-metier-d-entrepreneur-des-competencesa-developper-acquerir-et-maitriser.

8. Jonnaert, P. (2009). Compétences et socioconstructivisme un cadre théorique, Bruxelles, De Boeck.

9. Laviolette, E.M et Loué, C. (2006). Les compétences entrepreneuriales: définition et construction d'un référentiel. http://www.entrepreneuriat.com/uploads/media/laviolette loue2005 pdf. 25/02/2017

10. Lebrun, M. (2007, 2ème éd.). Théories et méthodes pédagogiques pour enseigner et apprendre. Bruxelles : De Boeck Université.

11. Le Boterf. (2010). Repenser la compétence, Editions Eyrolles.

12. Loué, C. et Barônet, J. (2015). Quelles compétences pour l'entrepreneur? Une étude de terrain pour élaborer un référentiel. Entreprendre \& Innover, 2015/4 ( $\left.\mathrm{n}^{\circ} 27\right)$.

13. Loué, C. et Majdouline, I. (2015). Les compétences de l'entrepreneur marocain : validation quantitative d'un référentiel, Revue Internationale PME, Volume 28, $\mathrm{N}^{\circ} 2$.

14. Omrane, A, Fayolle. A et Benslimane. O. (2011). Les compétences entrepreneuriales et le processus entrepreneurial: une approche dynamique. La Revue des Sciences de Gestion 2011/5 (n²51). 
15. Raynal, F. et Rieunier, A. (1997). Pédagogie : Dictionnaire des concepts clés. Paris : ESF.

16. Raucent, B. et Vander Borght, C. (2006). Etre enseignant: Magister? Metteur en scène ? Bruxelles : De Boeck Université.

17. Rege Colet, N. et Berthiaume, D. (2009). Savoir ou être? Savoirs et identités professionnels chez les enseignants universitaires, Dans R. Hofstetter \& B. Schneuwly (Eds). Savoirs en (trans)formation. Bruxelles : De Boeck Université.

18. Tardif, J. (2006). L'évaluation des compétences - documenter le parcours de développement. Montréal : Chenelière Education.

19. Toutain, O et Salgado, M. (2012). Comment améliorer la performance des pédagogies entrepreneuriales par la mise en action. $11^{\text {ème }}$ Congrès International Francophone en Entrepreneuriat et PME (CIFEPME), Oct 2012, BREST, France. pp.cd actes. <hal00842210>

20. Verzat et Fayolle. (2009). Pédagogie active et entrepreneuriat: Quelle place dans nos enseignements? Revue de l'entrepreneuriat, Volume $8, \mathrm{~N}^{\circ} 2$. 